\title{
THE PHARMACOKINETICS OF PENAMECILLIN
}

\author{
BY \\ M. GIBALDI AND M. A. SCHWARTZ \\ From The Department of Pharmaceutics, School of Pharmacy, State University of New York \\ at Buffalo, Buffalo, New York, U.S.A.
}

(Received September 22, 1966)

\begin{abstract}
Agersborg, Batchelor, Cambridge \& Rule (1966) have recently reported on the pharmacology of a new, semi-synthetic penicillin, penamecillin, in dogs. The compound is the acetoxymethyl ester of benzylpenicillin, which is rapidly hydrolysed to the latter by the action of non-specific esterases. There is considerable experimental evidence to indicate that no significant amount of the unchanged ester ever reaches the tissues after oral administration. Therefore, the intrinsic chemotherapeutic activity of the drug is identical to that of benzylpenicillin. However, penamecillin appears to offer some advantages over benzylpenicillin in that significantly more sustained blood levels are observed.
\end{abstract}

The purpose of this paper is to present a pharmacokinetic analysis of the absorption and elimination of penamecillin in order to provide an explanation for the prolonged blood levels observed after oral administration of this compound. The mathematical description of serum concentration versus time data is receiving increased attention by research pharmacists and pharmacologists. As noted by Wagner \& Nelson (1964), once data are described in mathematical terms they become subject to interpretation which must be consistent with the physiological reality of the system in vivo and the physicochemical and pharmaceutical properties of the drug.

\section{METHODS}

The mathematical analysis presented in this report was accomplished by using the data of Agersborg et al. (1966).

\section{RESULTS}

A comparison of the time course of mean serum levels of benzylpenicillin in 10 dogs following the oral administration of $25 \mathrm{mg} / \mathrm{kg}$ of penamecillin and potassium benzylpenicillin is shown in Fig. 1. The penamecillin data have been corrected from the original report to account for differences in mol. wt. It is apparent that the peak plasma level occurs more rapidly and is much higher after the administration of potassium benzylpenicillin. However, a serum level of benzylpenicillin greater than $0.1 \mu \mathrm{g} / \mathrm{ml}$. was maintained for $10 \mathrm{hr}$ after dosing with penamecillin as compared to the 5-6 hr duration provided by potassium benzylpenicillin. The serum concentration of $0.1 \mu \mathrm{g} / \mathrm{ml}$. was taken by Agersborg et al. (1966) as the minimum therapeutic level. 


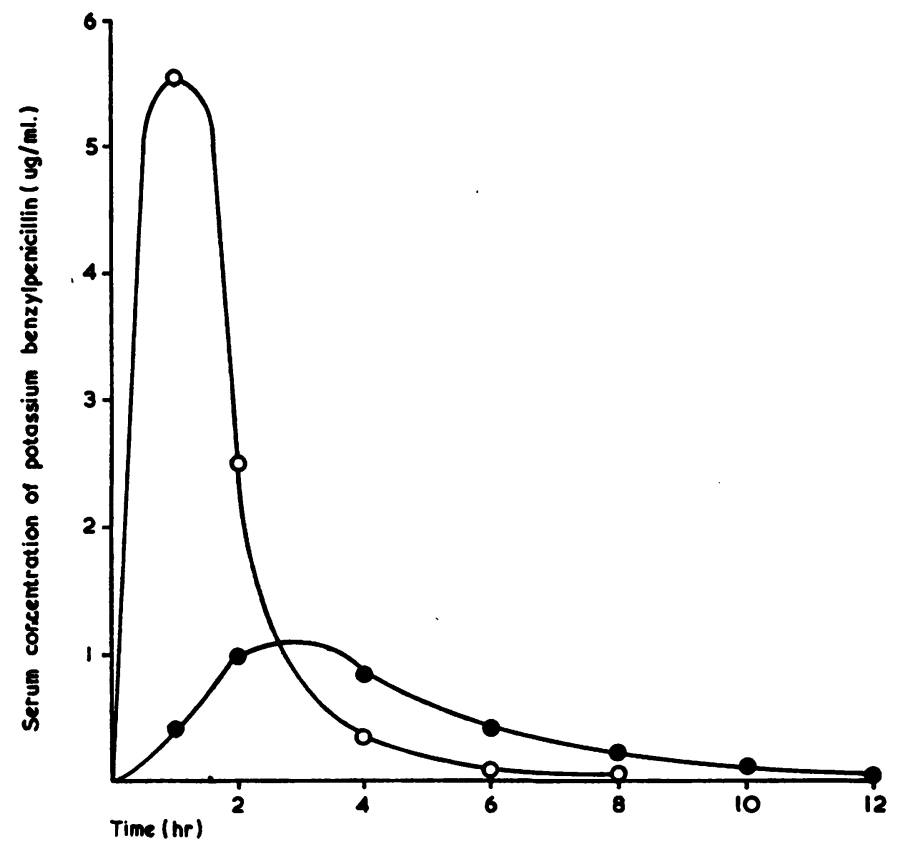

Fig. 1. Comparison of potassium benzylpenicillin serum concentration following a single oral dose of potassium benzylpenicillin $(O)$ and penamecillin $(\bullet)$.

Since inspection of the data suggested differences in the extent of absorption of the two compounds, the availability of penamecillin relative to benzylpenicillin was calculated. A general expression for physiological availability is

$$
\% \text { availability }=\frac{\text { amount absorbed after test dose }}{\begin{array}{c}
\text { amount absorbed following the same } \\
\text { dose in readily available form }
\end{array}}
$$

A number of methods are available to estimate physiological availability from blood level data (Wagner \& Nelson, 1964). Among these is the following relationship:

$$
\begin{aligned}
& \% \text { availability }=\int_{t=0}^{t=\infty} \begin{array}{c}
t \\
t=0
\end{array} \\
& \int_{t=0}^{t=\infty} \mathbf{C d t} \text { for readily available preparation }
\end{aligned}
$$

where $\int_{t=0}^{t=\infty} C d t$ is the area under the blood level-time plot for the respective preparations from $t=o$ to $t=\infty$. Estimation of area beneath a blood level versus time curve may be obtained by several methods. The method employed in this analysis involved the use of a planimeter. The areas under the curve (AUC) were calculated to be 11.4 and $5.3 \mathrm{mg}-\mathrm{hr} / \mathrm{ml}$. after administration of benzylpenicillin and penamecillin respectively.

If one makes the assumption that $100 \%$ of the administered dose of benzylpenicillin is absorbed then it may be calculated that $47 \%$ of the dose of penamecillin was absorbed. 
However, it is highly doubtful that benzylpenicillin is completely absorbed from the canine gastrointestinal tract. Studies in cats show that only one-third of an oral dose of benzylpenicillin is absorbed (McDermott, Bunn, Benoit, Dubois \& Reynolds, 1947). The reported oral availability of benzylpenicillin in man ranges from $10-30 \%$ (Free, Leonards, McCullagh \& Biro, 1944 ; Heatley, 1956). The oral availability of benzylpenicillin in dogs has not been reported in the literature but a reasonable approximation may be made on the basis of volume of distribution data. The apparent volume of distribution of benzylpenicillin in dogs was estimated from the data of Schachter (1948) by extrapolating the linear portion of a semilogarithmic plot of blood benzylpenicillin concentration versus time to the concentration axis and dividing the intercept into the dose administered intravenously. An apparent volume of $500 \mathrm{ml} . / \mathrm{kg}$ was calculated.

On the basis of this estimation, the fraction of benzylpenicillin absorbed in the studies of Agersborg et al. (1966) was estimated by using the following relationship (Wagner \& Nelson, 1964):

$$
\mathrm{V}=\frac{\mathrm{FD}}{\mathrm{K}(\mathrm{AUC})}
$$

where $\mathrm{D}$ is the oral dose, $\mathrm{F}$ is the fraction of dose absorbed, $\mathrm{K}$ is the first order elimination rate constant of benzylpenicillin, and AUC is the area under the blood level versus time curve. The elimination rate constant was determined from the slope of a semilogarithmic plot of concentration of benzylpenicillin in the blood versus time (Fig. 2) and found to be $0.87 \mathrm{hr}^{-1}$. The calculation of the elimination rate constant is discussed in greater detail below. By substituting the appropriate quantities into Eq. 3 the value for F was found to be $0.2-$ that is, $20 \%$ of the dose was absorbed-in good agreement with results observed in other species. Accordingly, it may be concluded that only about $10 \%$ of the administered dose of penamecillin is absorbed in the dog, and $90 \%$ is either degraded in the gastrointestinal tract or excreted unchanged in the faeces.

Despite the extremely poor absorption of penamecillin in the gastrointestistinal tract, the drug nevertheless exerts a chemotherapeutic effect for almost twice as long as benzylpenicillin. It was, therefore, of interest to evaluate the kinetic parameters of absorption and elimination. If one assumes that the rate of drug elimination adheres to first order kinetics, the following equations hold in the post-absorptive phase:

and

$$
\begin{aligned}
\ln C & =-K t+\ln C^{\circ} \\
K & =0.693 / t_{1}
\end{aligned}
$$

where $\mathrm{C}$ is the blood drug concentration at time $\mathrm{t}, \mathrm{C}^{0}$ is the hypothetical concentration at zero time, $\mathrm{K}$ is the first order elimination rate constant, and $t_{\frac{1}{2}}$ is the biological half life of the drug. Fig. 2 shows blood concentration of benzylpenicillin after equal oral doses of potassium benzylpenicillin and penamecillin, plotted as a function of time on semilogarithmic paper. The slope of the line obtained after administration of potassium benzylpenicillin yields an elimination rate constant of $0.87 \mathrm{hr}^{-1}$, corresponding to a biological half life of $0.8 \mathrm{hr}$. These values are in agreement with the findings of Schachter (1948). It is readily apparent from Fig. 2 that a significant difference exists in the slope of the two lines. From the linear portion of the curve obtained after administration of penamecillin an apparent elimination rate constant of $0.31 \mathrm{hr}^{-1}$ and a 


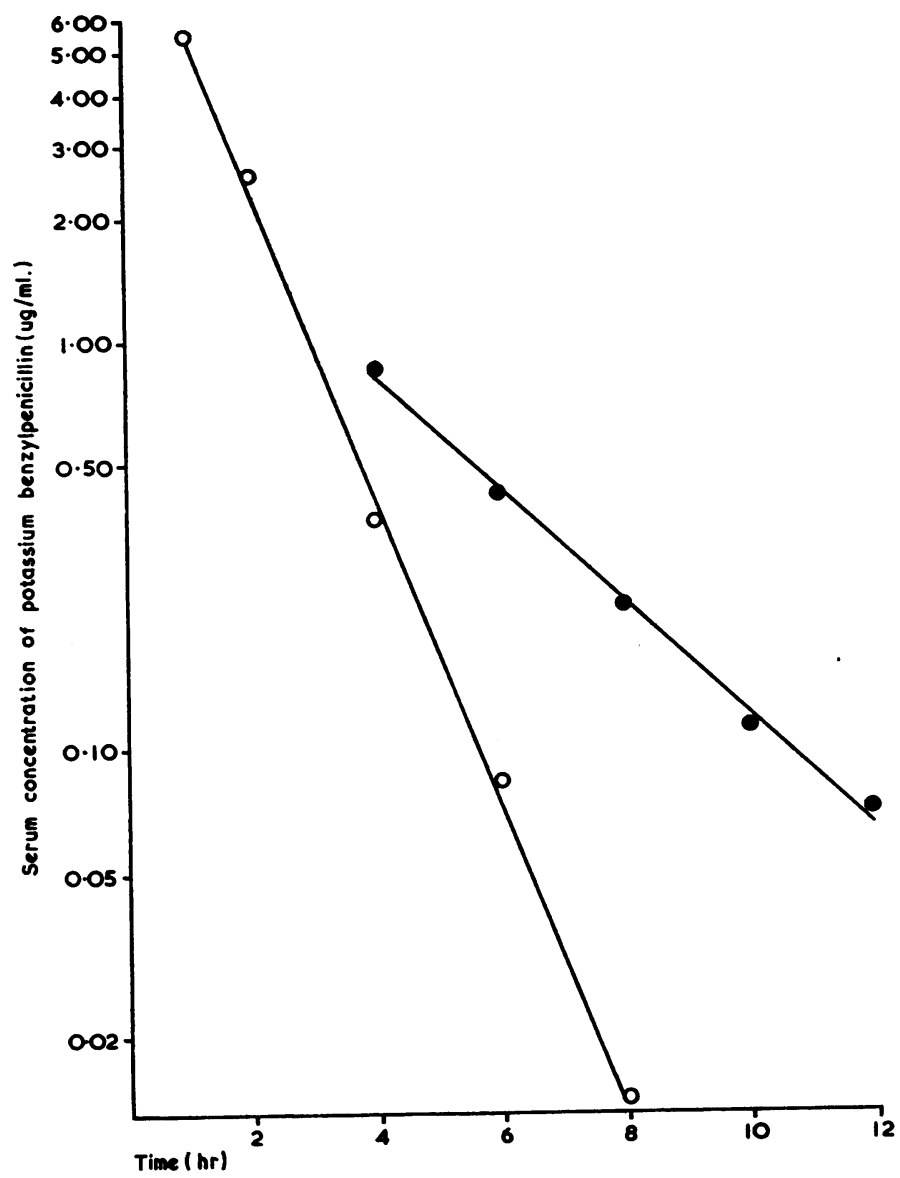

Fig. 2. Semilogarithmic plot of benzylpenicillin serum concentration versus time following equal oral doses of potassium benzylpenicillin $(O)$ and penamecillin $(\bullet)$.

corresponding biological half life of $2.2 \mathrm{hr}$ were calculated. Since only benzylpenicillin is present in the blood regardless of whether potassium benzylpenicillin or penamecillin is administered, the elimination rate constants should be identical. The shift in the elimination rate curve observed after administration of penamecillin suggests that this curve reflects both elimination and absorption and, therefore, is not post-absorptive data. It would appear that absorption of penamecillin occurs for some time, whereas benzylpenicillin is apparently no longer absorbed to any significant extent after $1 \mathrm{hr}$.

It is important to note that the excellent linearity observed after the administration of penamecillin provides an almost classic example of the danger in estimating biological half lives after oral administration of a drug which is poorly or slowly absorbed. Great care must be taken to prevent the introduction of error into calculations dependent on biological half life. Apparent half lives are nevertheless very valuable in that they provide a more realistic view of duration of activity. For example, the apparent half 
life of the drug rather than the true half life is the correct value to employ for determining dosage regimen. On this basis it would appear that a dose of penamecillin need be administered with about one-half the frequency of the same dose of potassium benzylpenicillin to obtain the same therapeutic effect.

The elimination data suggests that absorption of penamecillin is quite prolonged. Accordingly, the method of Wagner \& Nelson (1964) was applied to elucidate the absorption kinetics. The \% drug unabsorbed as a function of time is readily calculated by applying the following formula:

$$
\% \text { unabsorbed }=100-\% \text { absorbed }=100-\frac{C_{T}+K \int_{t=0}^{t=T} C d t}{K \quad \int_{t=0}^{t=\infty} C d t} \times 100
$$

where $C_{T}$ is the blood concentration at time $T$ and $K$ is the true elimination rate constant for benzylpenicillin. The integrals (areas under the curves) were calculated as previously described. A semilogarithmic plot of \% penamecillin unabsorbed (based on the total amount of drug ultimately absorbed) versus time is shown in Fig. 3. After an intial lag, absorption rate is seen to follow closely apparent first order kinetics with a rate constant of $0.34 \mathrm{hr}^{-1}$ and a half life of $2.05 \mathrm{hr}$. It is clear that absorption of penamecillin occurs over a 10-hr period as contrasted to potassium benzylpenicillin, which is apparently not absorbed to any significant extent beyond one hour after administration.

The mathematical analysis permits the conclusion that the prolonged duration of activity is a function of the slow, continual absorption of penamecillin (or its hydrolysis product, benzylpenicillin) throughout the gastrointestinal tract. A number of striking differences appear to exist in the absorption characteristics of orally administered potassium benzylpenicillin and penamecillin. Potassium benzylpenicillin is absorbed after administration apparently from both the stomach and duodenum. There is no doubt that some benzylpenicillin is hydrolysed to inactive products in the stomach but this alone does not appear to account for the fact that $80 \%$ of the dose is unabsorbed. The poor absorption of intact benzylpenicillin in the lower intestine may be due to the low $\mathrm{pK}_{\mathrm{a}}$ of the drug and the fact that it is toally dissociated at $\mathrm{pH} 5-6$.

Penamecillin, a neutral molecule, appears to be very poorly absorbed in the stomach. As suggested by Agersborg et al. (1966), the ester grouping has some protective effect against the degradation of the benzylpenicillin moiety in the acid medium of the stomach, and the possibility exists that some unchanged penamecillin is absorbed in this region. This would be readily hydrolysed to benzylpenicillin by esterases in the mucosal cells or serum. The solubility of penamecillin in water at $25^{\circ} \mathrm{C}$ has been determined spectrophotometrically in our laboratories to be about $4.5 \mathrm{mg} / 100 \mathrm{ml}$. The low solubility of the unchanged compound would tend to produce a low dissolution rate and thereby limit the amount of penamecillin absorbed as the intact molecule. Poor gastric absorption would also serve to explain the lag time observed in the \% drug unabsorbed versus time plot (Fig. 3).

Agersborg et al. (1966) have further proposed that once penamecillin reaches the duodenum rapid hydrolysis can take place in the intestinal lumen by the action of pancreatic lipase. According to our findings it would not appear that hydrolysis is rapid, 


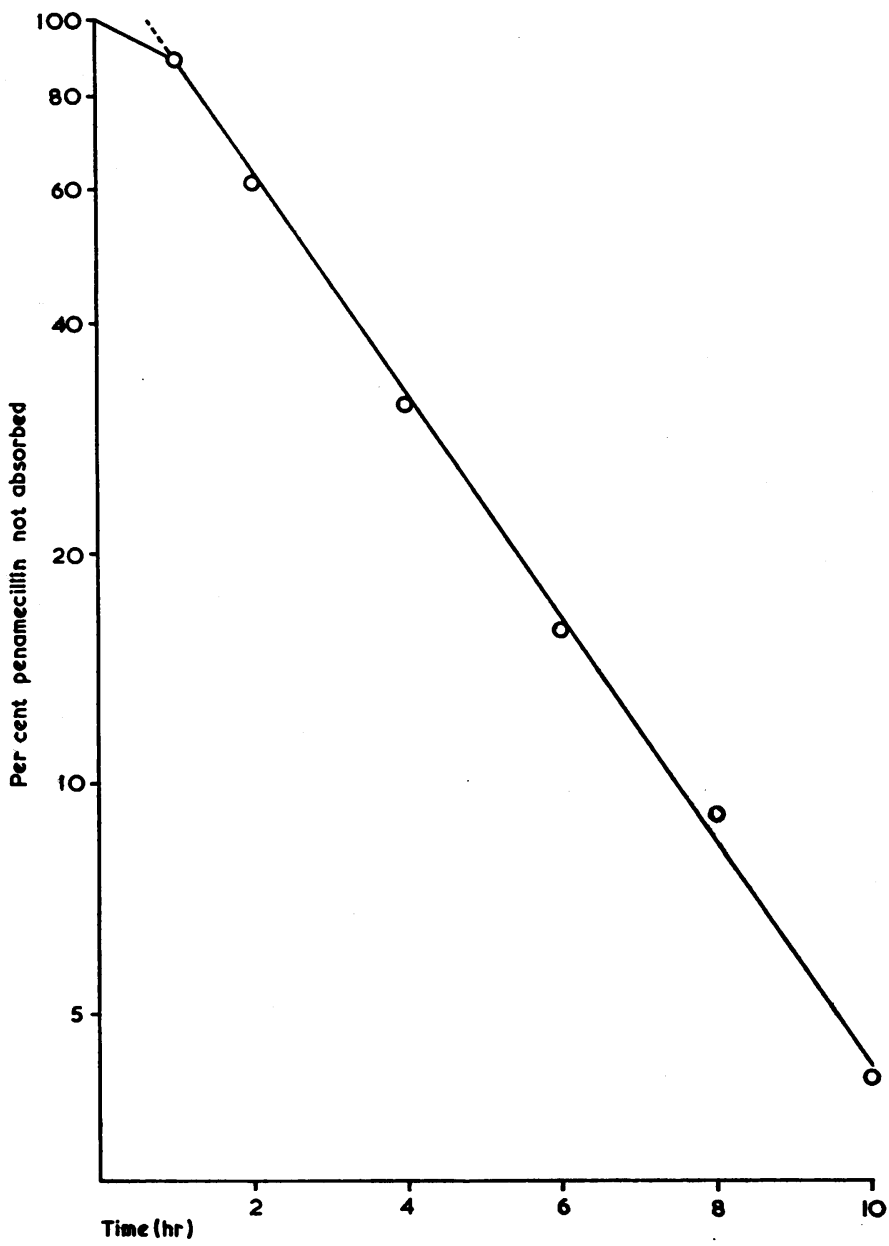

Fig. 3. Plot of \% penamecillin unabsorbed (logarithmic scale, based on total amount of drug ultimately absorbed) versus time.

considering that the total turnover of penamecillin is only about $10 \%$ of the dose. Moreover, the absorption kinetics suggest that penamecillin is absorbed as the intact molecule both in the duodenum and in the lower intestine. The prolonged serum levels clearly indicate that absorption occurs throughout the length of the intestine. In view of the apparent lack of absorption of benzylpenicillin beyond the duodenum, it is unlikely that the absorption of penamecillin in the lower intestine is attributable to prior conversion to benzylpenicillin. It is of interest to note that Lin, Guarini, Eriksen \& Swintosky (1964) have reported poor availability of benzylpenicillin from the distal portions of the dog intestine. This report confirms our theoretical conclusions. Accordingly, it is proposed that the prolonged benzylpenicillin serum levels observed after the administration of penamecillin are essentially due to absorption of unchanged penamecillin throughout the gastrointestinal tract and rapid conversion by mucosal tissue and serum esterases to benzylpenicillin. 


\section{SUMMARY}

1. The kinetics of absorption and elimination in the dog of a new penicillin derivative, penamecillin, have been determined from data in the literature.

2. Calculations of physiological availability indicate that only about $10 \%$ of a $25 \mathrm{mg} / \mathrm{kg}$ oral dose of penamecillin is absorbed in the dog.

3. The absorption of penamecillin is slow and prolonged. The rate of appearance of benzylpenicillin in the blood after an oral dose of penamecillin was apparent first order with a half life of about $2 \mathrm{hr}$.

4. It is proposed that the prolonged benzylpenicillin serum levels observed after administration of penamecillin are chiefly a function of absorption of unchanged penamecillin throughout the intestinal tract.

\section{REFERENCES}

Agersborg, H. P. K., Batchelor, A., CAmbridge, G. W. \& Rule, A. W. (1966). The pharmacology of penamecillin. Br. J. Pharmac. Chemother., 26, 649-655.

Free, A. H., Leonards, J. R., McCullaGh, D. R. \& BiRo, B. E. (1944). Urinary excretion of penicillin after oral administration to normal subjects. Science, N.Y., 100, 431-432.

HEATLEY, N. G. (1956). Comparative serum concentration and excretion experiments with benzyl penicillin (G) and phenoxymethyl penicillin (V) on a single subject. Antibiotic Med., 2, 33-41.

Lin, T. H., GuARINI, J. R., ERIKSEn, S. P. \& SwIntoskY, J. V. (1964). Effect of site of release on the absorption of trimeprazine-S $S^{35}$ and penicillin $G$ in dogs. J. Pharm. Sci., 53, 1357-1359.

MCDeRmott, W., BunN, P. A., BENoIt, M., DuBors, R. \& ReYnolds, M. E. (1946). Absorption, excretion and destruction of orally administered penicillin. J. Clin. Invest., 25, 190-210.

SCHACHTER, R. J. (1948). Fate and distribution of penicillin in the body. I, Circulation of penicillin in lymph. Proc. Soc. exp. Biol. Med., 68, 29-34.

WAGNER, J. G. \& NeLSON, E. (1964). Kinetic analysis of blood levels and urinary excretion in the absorptive phase after single doses of drug. J. pharm. Sci., 53, 1392-1403. 